\title{
Preface
}

This Symposium was the first IAU Symposium devoted to extra-solar planets, and the papers presented reflected the rapid growth of the field. The idea for the Symposium originated within the IAU itself, as one of the Symposia to be held during the XXIV General Assembly in Manchester, England in August 2000. The extra-solar planet community took on the task of organising the meeting. It was decided to have a slightly unusual format, with the talks being almost all one-hour Invited Reviews, and nearly all contributed papers being posters. This format was afterwards generally agreed to be very effective. The success of the meeting was to a large extent due to the high quality of the presentations by the speakers. Forming part of the General Assembly, there was no specific list of attenders, but the large lecture theatre was for all four days packed to overflowing. The meeting was divided into five themes: I. Discovery and study of extrasolar planets - current ; II. Progress in the theory of planet formation ; III. Structure and evolution of planets ; IV. Protoplanetary and Beta Pic disks ; V. Discovery and study of extrasolar planets - future. In this volume the papers are classed into these five themes, with the Invited Reviews first, and then the poster papers (in alphabetical order of first author).

The papers are substantially as they were when they were submitted before March 1st, 2001.

Alan Penny, Pawel Artymowicz, Anne-Marie Lagrange, and Sara Russell Editors 


\section{Scientific Organising Committee}

Roger Angel (U Arizona, USA)

Immo Appenzeller (U Heidelberg, Germany )

Pawel Artymowicz (Stockholm Obs., Sweden)

Alan Boss ( CIW DTM, USA)

Adam Burrows (U Arizona. USA)

Daniel Gautier (Meudon, France)

Anne-Marie Lagrange (U Grenoble, France )

Michel Mayor (Geneva Obs., Switzerland)

Y. Nakagawa Japan)

Alan Penny ( RAL, UK) (Chair)

Sara Russell (NHM, UK)

Penny Sackett (Kapteyn Institute, Netherlands)

Günther Wuchterl (MPE, Germany)

\section{Acknowledgements}

This meeting was made possible by the enthusiam of the IAU, and also by the Local Organising Committee of the XXIV General Assembly in Manchester, who provided all the hosting arrangements. These Proceedings would not have emerged without the persistence and support of the IAU.

\section{The Editors}

Alan Penny, Rutherford Appleton Laboratory, UK Pawel Artymowicz, Stockholm Observatory, Sweden Anne-Marie Lagrange, U Grenoble, France

Sara Russell, Natural History Museum, UK 